\title{
Nanometer lithography on silicon and hydrogenated amorphous silicon with low energy electrons
}

\author{
N. Kramer, ${ }^{\text {a) }}$ J. Jorritsma, ${ }^{\text {a) }}$ H. Birk, and C. Schönenberger \\ Philips Research, Prof. Holstlaan 4. 5656 AA Eindhoven, The Netherlands
}

(Received 22 August 1994; accepted 16 March 1995)

\begin{abstract}
The oxidation of a hydrogen terminated Si surface can locally be induced with a scanning tunnelling microscope (STM) operating in air or with a beam of free electrons in a controlled oxygen environment. The oxidation mechanism of both processes was studied and compared. The oxidation with the STM in air depends strongly on the applied tip-substrate voltage and writing speed, but is not proportional to the tunnelling current. This is in contrast to the process with a beam of free electrons. The thickness of the electron beam induced oxide is studied as a function of electron energy, electron dose, and oxygen pressure. Oxide thicknesses of $0.5-3 \mathrm{~nm}$ are measured using Auger spectroscopy. The initial step of the oxidation process is the electron beam induced removal of hydrogen from the surface. The electron dose requirement for this step was determined as a function of electron energy. The dose is found to be minimal for $100 \mathrm{eV}$ electrons, and is $\approx 4$ $\mathrm{mC} / \mathrm{cm}^{2}$. Oxide lines made with the $\mathrm{STM}$ on $\mathrm{Si}(110)$ were used as a mask to wet etch the pattern into the $\mathrm{Si}(110)$. With tetramethyl ammonium hydroxide, a selective anisotropic etch liquid, trenches with a width of $35 \mathrm{~nm}$ and a depth of $300 \mathrm{~nm}$ were made. We show that it is also possible to locally oxidize hydrogenated amorphous silicon $(a-\mathrm{Si}: \mathrm{H})$ and use the oxide as an etching mask. Hydrogenated amorphous silicon has the advantage that it can be deposited in very thin layers on almost any substrate and therefore has great potential as STM and electron-beam resist. () 1995 American Vacuum Society.
\end{abstract}

\section{INTRODUCTION}

Further down scaling of device dimensions for IC technology or for the studying of new physical phenomena demands lithographic technologies that allow the fabrication of structures with nanometer dimensions. Electron-beam $(e-$ beam) lithography is the standard technique to fabricate nanostructures. Typical electron energies are in the range of $10-50 \mathrm{keV}$. The resolution is limited by proximity effects, caused by scattered electrons. In order to avoid proximity effects many groups are investigating alternative high resolution lithographic processes based on low energy electrons. ${ }^{1-5}$ The process of local oxidation of hydrogen passivated silicon (100) and (111) with a scanning tunnelling microscope (STM) is very promising. ${ }^{2-5}$ A negative tipsample voltage induces the removal of hydrogen, and leads to the oxidation of the surface. It has been demonstrated that with this technique, oxide lines with a width of $\approx 2 \mathrm{~nm}$ can be made in ultrahigh vacuum (UHV). ${ }^{3}$ In air oxide lines of $25 \mathrm{~nm}$ have been fabricated and used as a mask for wet etching. ${ }^{4}$ As the written oxide masks are very thin $(\approx 1 \mathrm{~nm})$, deep structures are difficult to realize. To enable the fabrication of deep structures we studied alternative etching liquids and used $\mathrm{Si}(110)$ instead of $\mathrm{Si}(100)$ or $\mathrm{Si}(111)$. We were able to fabricate trenches with a width of $\approx 35 \mathrm{~nm}$ and a depth of $\approx 300 \mathrm{~nm}$. Interesting applications can be thought of for this type of structure, for example metal evaporation under an angle, yielding narrow metal wires for electrical transport measurements. A drawback of this technique is the requirement of crystalline silicon as substrate, in order to achieve stable hydrogen passivation. We show that the principle of

a) Also at The University of Twente, Enschede, The Netherlands.
STM induced oxidation also works on a very thin film $(25$ $\mathrm{nm})$ of hydrogenated amorphous silicon $(a-\mathrm{Si}: \mathrm{H})$. Oxide patterns were made with the STM on $a-\mathrm{Si}: \mathrm{H}$ and successfully used as etch mask. $a$-Si:H has the advantage that very thin films can be deposited on almost any substrate and that the films are stable against oxidation in air. This makes $a-\mathrm{Si}: \mathrm{H}$ widely applicable as a resist.

In UHV the STM has been used to remove the hydrogen from a hydrogen passivated silicon surface; consecutively the depassivated areas could be oxidized by introducing oxygen. ${ }^{3}$ The advantage of using an UHV STM is that ultra sharp tips can be used, which give higher resolution than the tips used in air. If a negative voltage $(10-100 \mathrm{~V})$ is applied to these tips, low energy electrons are emitted, which causes the removal of hydrogen from the Si surface. To investigate the mechanism of e-beam induced oxidation, we built an UHV setup that allows us to expose a $\mathrm{Si}$ surface to low energy electrons in UHV or in a controlled oxygen environment. The dose requirements for the hydrogen removal were determined. The dependence of oxide thickness on the electron energy, electron dose, and oxygen pressure was studied with a scanning Auger microscope. The oxidation mechanisms with an e beam and with the STM in air are compared; experiments show that the mechanisms are different.

\section{EXPERIMENT}

Crystalline $\mathrm{Si}(110)$ and $\mathrm{Si}(100)$ substrates or $a-\mathrm{Si}: \mathrm{H}$ films deposited on $\mathrm{Si}(100)$ were used in the experiments with the STM and the UHV e beam. The $\mathrm{Si}(110)$ and $\mathrm{Si}(100)$ (both $n$-type, $10 \mathrm{~m} \Omega \mathrm{cm}$ ) substrates were cleaned with trichloroethylene and acetone and rinsed in ethanol, before dipping them for $30 \mathrm{~s}$ in dilute hydrofluoric acid (10\%) for the hy- 
drogen passivation, followed by a $10 \mathrm{~s}$ rinse in deionized water. The thin films $(25 \mathrm{~nm})$ of $n$-type hydrogenated amorphous silicon (phosphorous doped, $\sim 10^{20}-10^{21} \mathrm{~cm}^{-3}$ ) were deposited on undoped $\mathrm{Si}(100)$ in a plasma enhanced chemical vapor deposition (PECVD) system (substrate temperature $200{ }^{\circ} \mathrm{C}$ ). Before writing oxide lines on $a$-Si:H, the sample was dipped in HF (10 s) to remove any native oxide.

For the STM oxidation we used an instrument that operated in air. The experiments were performed with mechanically prepared PtRd tips or with commercial PtIr tips (from Materials Analytical Services). Oxide lines were written with negative tip-substrate voltages $U_{t}=-2.5$ to $-4.0 \mathrm{~V}$. Imaging and writing were done in the constant current mode with tunnelling currents ranging from 2 to $100 \mathrm{pA}$. The writing speed is varied from 0.1 to $1 \mu \mathrm{m} / \mathrm{s}$. After writing with negative $U_{t}$, we sometimes imaged the written pattern with the STM using a positive tip-substrate voltage $\left(U_{t}=+2 \mathrm{~V}\right.$, tunnelling current $5 \mathrm{pA}$ ). Because of the reduced conductivity on oxidized $\mathrm{Si}$ areas, the oxide lines appear dark in STM images.

Imaging with the STM is possible because the rate of oxidation with positive $U_{t}$ is much lower than with negative $U_{t}$. However, extensive imaging oxidizes the surface to such an extent that the following etching process is no longer reproducible. To avoid this problem, oxide lines made for etching experiments were not imaged by the STM. Following the exposure the pattern is transferred into the substrate by wet etching. To fabricate narrow and deep structures it is necessary to have a very selective and anisotropic etch liquid. We used three etching liquids: $\mathrm{KOH},{ }^{6} \mathrm{EDP}$ (ethylendiamin pyrocatecol), ${ }^{7}$ and TMAH (tetramethyl ammonium hydroxide). ${ }^{8}$ All three liquids etch anisotropically, with a very low etch rate for the $\operatorname{Si}\{111\}$ planes with respect to the $\{110\}$ and $\{100\}$ planes. For example, KOH etches Si(111) 300 times slower than $\mathrm{Si}(100)$; EDP and TMAH etches $\mathrm{Si}(111) 10$ times slower than $\mathrm{Si}(100)$. Anisotropically etched structures are bounded by $\{111\}$ crystal planes, the planes which are etched at the slowest rate. Since the angle between the (100) and the (111) planes is $55^{\circ}$, the sidewalls of the etched trenches in $\mathrm{Si}(100)$ are inclined by $55^{\circ}$ with respect to the surface. This limits the achievable depth of trenches. Deeper trenches can be fabricated in $\operatorname{Si}(110) .{ }^{6}$ In this case the sidewalls of the trenches will be perpendicular to the surface, if the oxide lines are written along the [1I2] direction. The depth of the trench is then determined by the etching selectivity, i.e., silicon-to-silicon-oxide etch rate ratio. $\mathrm{KOH}$ etches most anisotropically, but is not as selective as EDP or TMAH. With TMAH and EDP the selectivity can be improved by a factor of 10 over $\mathrm{KOH}$. In the case of EDP the quality of the etched surface is poor due to residues. Residue free etching was only possible at temperatures $>100{ }^{\circ} \mathrm{C}$. At these temperatures the etch rate is too large $(>1.0 \mu \mathrm{m} / \mathrm{min})$ to be practical for nanometer scale structures. Since TMAH etches residue free, even at low etching rates $(0.1-0.6 \mu \mathrm{m} /$ min), we have found this liquid to be most convenient for our experiments. Although not as anisotropic as $\mathrm{KOH}$, this etchant is very selective: 1 monolayer of silicon oxide can withstand $1 \mu \mathrm{m}$ deep etching in $\mathrm{Si}(110){ }^{8}$

We used 40 wt. \% TMAH solution at $75^{\circ} \mathrm{C}$ for the etch- ing of $\mathrm{Si}(110)$ (etching rate $\sim 0.6 \mu \mathrm{m} / \mathrm{min}$ ) and the same solution at $60{ }^{\circ} \mathrm{C}$ for the etching of $a$-Si:H (etching rate $\sim 0.2$ $\mu \mathrm{m} / \mathrm{min})$.

To study the electron-beam induced oxidation of $\mathrm{Si}$, a dedicated UHV system was built. In this setup a sample can be exposed to e-beam radiation in a controlled oxygen environment. The electron gun system is differentially pumped, with the e beam focused through a hole (diameter $30 \mu \mathrm{m}$ ). The electron-beam current is calibrated with a Faraday cup located on the sample holder. The electron energy $(20 \mathrm{eV}-1$ $\mathrm{keV}$ ) was varied by applying a retarding voltage to the sample. The base pressure of the sample chamber is in the $10^{-10}$ Torr range. The sample can be introduced via a load lock; it requires $5 \mathrm{~min}$ to move a sample from air into the vacuum chamber.

Two types of experiments were performed, in the first experiment the dose for the removal of hydrogen atoms from the hydrogen terminated $\mathrm{Si}(100)$ surface was determined. An HF dipped $\mathrm{Si}(100)$ sample was exposed in UHV to electrons of varying electron energies and exposure times. After the different exposures, done at different locations on the sample, the sample is taken out of the chamber. If hydrogen has been removed, oxidation will occur instantly when bringing the sample in air, forming a thin oxide layer. ${ }^{9}$ The sample is then etched with TMAH at $75{ }^{\circ} \mathrm{C}$ for $10 \mathrm{~s}$. This etches about $100 \mathrm{~nm}$ of $\mathrm{Si}$, but does not etch through the formed oxide layer. Inspection of the etched sample with a scanning electron microscope (SEM) reveals the exposure requirements for the removal of hydrogen as a function of the electron energy.

In the second type of experiments a hydrogen terminated $\mathrm{Si}(100)$ surface is exposed to electrons in an oxygen environment. The oxide thickness is determined as a function of electron energy $(20 \mathrm{eV}-1.0 \mathrm{keV})$, electron dose (0.1-1.5 $\mathrm{C} / \mathrm{cm}^{2}$; achieved by using a beam current of $40 \mathrm{nA}$ with varying exposure times $0.5-6 \mathrm{~min}$ ) and oxygen pressure $\left(10^{-7}-10^{-4}\right.$ Torr $)$. We estimated the oxide thickness from Auger spectra, measured with a Perkin-Elmer PHI 600 scanning Auger microscope (SAM). The $\mathrm{Si}(L M M)$ peak is known to chemically shift in energy if measured on a $\mathrm{SiO}_{2}$ film. The ratio $R$ of the peak intensities of the shifted peak with respect to the unshifted $\mathrm{Si}$ peak, due to the bulk $\mathrm{Si}$ below the thin $\mathrm{SiO}_{2}$ film, depends in a phenomenological manner on the oxide thickness $d_{\text {oxide }}$ according to ${ }^{10}$

$$
d_{\text {oxide }}=\lambda \times 0.743 \times \ln \left(\frac{R}{A}+1\right) .
$$

$A$ is the ratio of the $\mathrm{Si}(L M M)$ peak intensities from bulk $\mathrm{SiO}_{2}$ and from bulk $\mathrm{Si}$ and is measured to be 0.091 , using a clean $\mathrm{Si}$ sample and a sample with a $\approx 100 \mathrm{~nm}$ thick $\mathrm{SiO}_{2}$ layer. The escape depth $\lambda$ for these Auger electrons was estimated in the following way: $\mathrm{A} \mathrm{Si}(100)$ sample was given an UV ozone treatment for $6 \mathrm{~min}$. After this the native oxide thickness was measured with a spectroscopic ellipsometer. This gave a thickness of $1.6 \pm 0.2 \mathrm{~nm}$. Directly after the ellipsometer measurement the Auger spectrum was measured for this sample. From this spectrum $R$ was obtained, and together with the thickness measured with ellipsometry it 
was used to calculate $\lambda$. This resulted in an escape depth of 7.6 ̊, which is consistent with data from the literature 5-15 $\AA^{11}$

The transfer of the sample from the electron-beam writing system through air to the UHV scanning Auger microscope takes approximately $5 \mathrm{~min}$. Since the rate of oxidation in air is slow after the formation of the first silicon oxide monolayer, we expect little additional oxide growth on the areas that were oxidized with the electron beam to a thickness of a few nanometers. We estimate that the systematic error, due to the transfer in air, in the measured oxide to be $\approx 0.1 \mathrm{~nm}$. This is smaller than the accuracy of the method used to calculate the oxide thickness $( \pm 0.3 \mathrm{~nm})$.

\section{RESULTS AND DISCUSSION}

\section{A. STM lithography}

In this section we discuss the experimental results of the oxidation of Si with the STM in air. The oxidation mechanism was studied by varying three important parameters; tip-sample voltage, tunnelling current, and writing speed.

Figure 1 shows STM images of oxide lines made for different writing parameters. All lines are written with a double pass; only the top part of the images should be considered, because close to the bottom the lines do not exactly overlap due to piezo creep effects. Figure 1(a) shows oxide lines written with different tip-sample voltages $U_{t}$ ranging from -2.5 to $-4.0 \mathrm{~V}$. The oxide lines appear dark in the STM images because of the reduced conductivity on the oxidized areas. To maintain a constant current, the electronic feedback system decreases the tip-sample distance. This results in a black contrast in the STM image. The oxidation is more pronounced (darker oxide lines) for larger negative voltages. For $U_{t}>-2.0 \mathrm{~V}$ no clear oxide lines are formed for this writing speed. In a simple picture, the electrons from the tip induce the removal of hydrogen from the surface, which leads to the oxidation of the silicon. A threshold energy of $2.5 \mathrm{eV}$ for the electrons is required to break the $\mathrm{Si}-\mathrm{H}$ bond and induce the oxidation reaction. Increasing the current would increase the efficiency of the process. Figure 1(b) shows oxide lines, written at constant speed $(1 \mu \mathrm{m} / \mathrm{s})$ and fixed voltage $U_{t}=-3.5 \mathrm{~V}$ ), with tunnelling currents ranging from 2 to $100 \mathrm{pA}$. Increasing the current by a factor of 50 hardly influences the oxidation. This is in contrast to the experiments in UHV where we find a clear dose dependence for the oxidation process (shown in Sec. III B). The writing speed has a profound effect on the oxidation, as can be seen in Fig. 1(c). Decreasing the writing speed to 8 s/line increases the oxidation significantly. Because of the independence of the oxide formation on the tunnelling current [Fig. $1(b)]$, the simple picture of electron induced removal of hydrogen from the surface cannot be correct. Figure 1 demonstrates that the oxidation process depends on the voltage and the writing speed, but that it does not depend on the tunnelling current. The voltage dependence is in agreement with the electrical field induced oxidation process proposed recently, ${ }^{5}$ because the electrical field between the tip and a heavily doped $\mathrm{Si}$ sample is approximately the $U_{t}$ divided by the tip to sample distance. Figure 2 shows SEM pictures of a
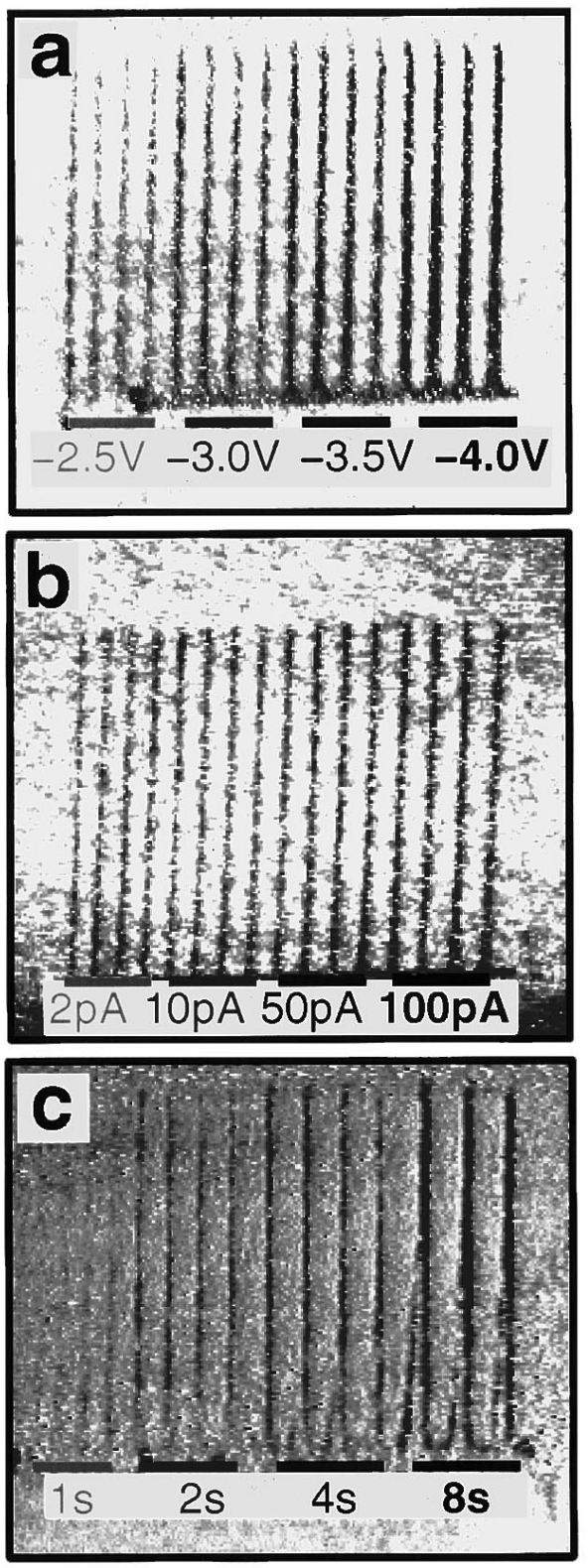

FIG. 1. (a) STM image (1.5-1.5 $\left.\mu \mathrm{m}^{2}\right)$ of oxide lines written with different tip-sample voltages (current, 2 pA; speed, 2 s/line). (b) STM image (1.5$1.5 \mu \mathrm{m}^{2}$ ) of oxide lines written with different tunnelling currents (voltage, $-3.5 \mathrm{~V}$; speed, $1 \mathrm{~s} /$ line). (c) STM image $\left(1.5-1.5 \mu \mathrm{m}^{2}\right)$ of oxide lines written with different writing speeds (voltage, $-3.5 \mathrm{~V}$; current, $2 \mathrm{pA}$ ).

line pattern after wet etching, for the case of oxide lines written along the [1T2] direction on $\mathrm{Si}(110)$. Figure 2(a) demonstrates that roughly $300 \mathrm{~nm}$ of Si can be etched without significant under etching of the oxide lines. The oxide lines are $90 \mathrm{~nm}$ wide and $35 \mathrm{~nm}$ apart from each other [Fig. 2(b)]. Thus writing along the [112] direction on $\mathrm{Si}(110)$ and using the anisotropic and selective etch liquid TMAH make it possible to produce $35 \mathrm{~nm}$ wide trenches with an aspect ratio of about 10 . The principle of local oxidation only works if the Si surface is properly terminated with hydrogen. In an attempt to structure GaAs, Snow, Campbell, and Shanabrook ${ }^{4}$ have grown a thin film of $\mathrm{Si}(100)$ epitaxially on GaAs and passivated it with hydrogen. The $\mathrm{Si}$ was oxidized with the STM and the oxide pattern could be transferred into 

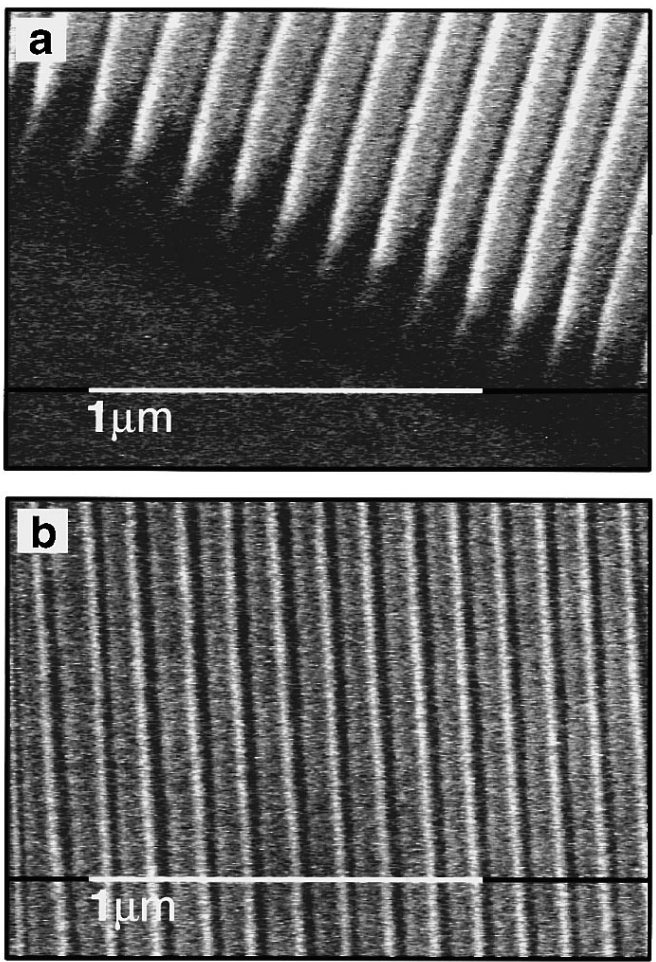

FIG. 2. SEM picture of STM written oxide lines on $\mathrm{Si}(110)$ after etching with TMAH. Oxide lines were written with $U_{t}=-3.5 \mathrm{~V}$, current of $5 \mathrm{pA}$, and speed of $0.5 \mu \mathrm{m} / \mathrm{s}$. In (a) the sample is tilted by $60^{\circ}$ SEM imaging.

the GaAs by wet etching. This procedure, however, is restricted to a limited number of substrates on which $\mathrm{Si}$ can be grown epitaxially. It could be extended to other substrates if a thin film with a stable hydrogen passivation can be grown. Hydrogenated amorphous silicon $(a-\mathrm{Si}: \mathrm{H})$ is a material that satisfies these conditions. ${ }^{12}$ With the STM we successfully oxidized the surface of a $25 \mathrm{~nm} a-\mathrm{Si}: \mathrm{H}$ film and used the oxide pattern as an etching mask. Figure 3 shows a SEM picture of $50 \mathrm{~nm}$ lines after etching $25 \mathrm{~nm} a-\mathrm{Si}: \mathrm{H}$ and $25 \mathrm{~nm}$ $\mathrm{Si}(100)$ with TMAH. A drawback of $a-\mathrm{Si}: \mathrm{H}$ is that anisotropic etching is impossible. High resolution can only be obtained with very thin films. Our preliminary results show

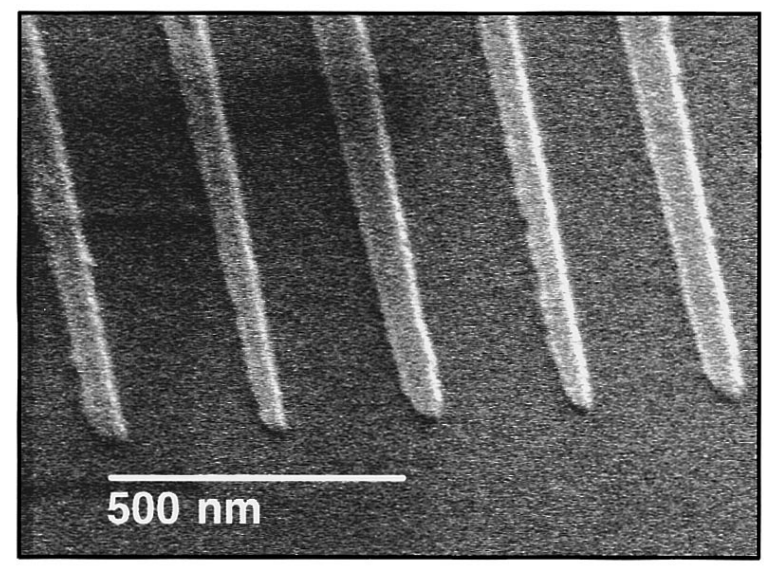

FIG. 3. SEM picture of STM written oxide lines on a thin $a-\mathrm{Si}: \mathrm{H}$ film after etching with TMAH. Oxide lines were written with $U_{t}=-3.5 \mathrm{~V}$, current of $5 \mathrm{pA}$, and speed of $1.0 \mu \mathrm{m} / \mathrm{s}$.

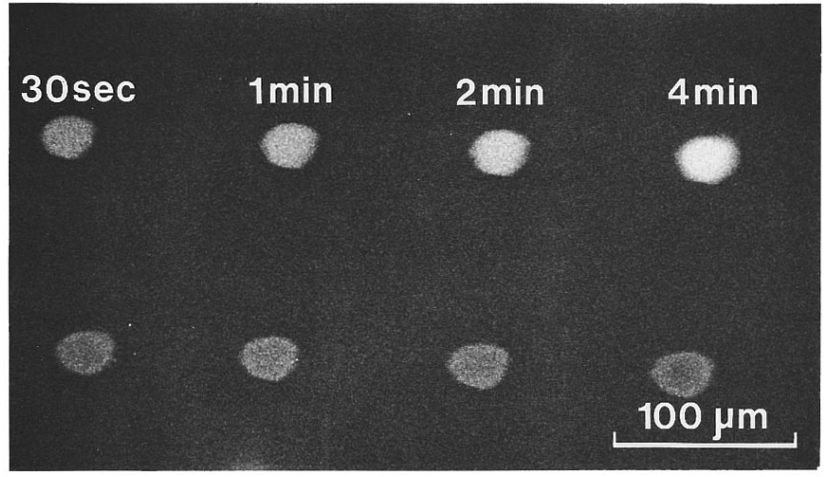

FIG. 4. SEM picture of oxide dots made with an electron beam (1.5 keV) in an oxygen environment of $10^{-4}$ Torr (top row) and in UHV (bottom row) for different exposure times (as indicated in the picture), with beam current of $40 \mathrm{nA}$.

that it is possible to structure a thin $(20 \mathrm{~nm})$ metal (TaIr) film using a $a-\mathrm{Si}: \mathrm{H}$ resist layer deposited on the metal. The oxide pattern, written with the STM, remains on the metal film after etching the $a-\mathrm{Si}: \mathrm{H}$. The remaining oxide pattern is transferred into the metal film by ion milling.

\section{B. Electron-Beam Induced Oxidation}

Figure 4 shows a SEM picture of oxide dots on $\mathrm{Si}(100)$ made with an electron beam in an oxygen environment with a pressure of $10^{-4}$ Torr (top row in Fig. 4) and in UHV (bottom row in Fig. 4) for different doses (exposure times of $30 \mathrm{~s}$ to $4 \mathrm{~min}$; beam current of $40 \mathrm{nA}$ ). The secondary electron emission from $\mathrm{SiO}_{2}$ is three times larger than from $\mathrm{Si}$, which is the reason why oxidized areas appear bright. The brighter an oxide dot appears in the SEM image, the thicker the oxide is. From this picture it is clear that the oxide thickness increases with increasing dose for electron exposure in an oxygen environment. In contrast, the dots made in UHV (no oxygen present during exposure) do not show a dose dependence. In this case oxidation occurs if the sample is removed from the UHV chamber into the air, at places where the hydrogen is removed from the surface. In Fig. 5 the re-

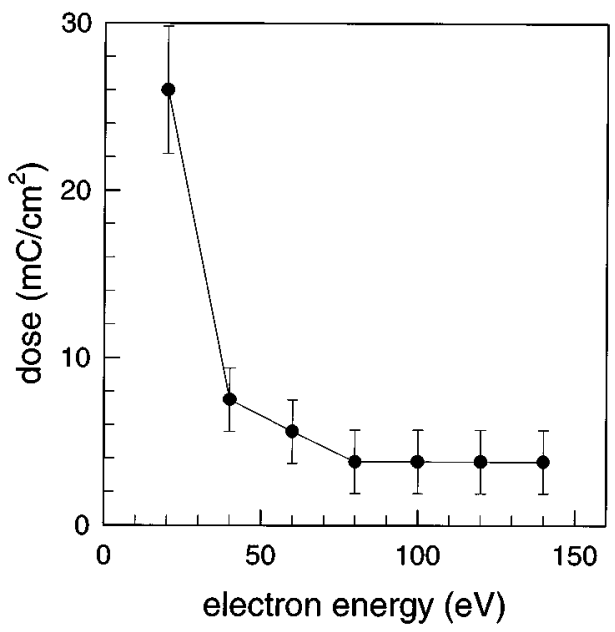

FIG. 5. Electron dose required to remove the hydrogen from the hydrogen terminated silicon surface in UHV as function of electron energy. 
quired dose to remove the hydrogen from the surface is plotted as a function of incident electron energy $E_{p}$. For $E_{p}=80-140 \mathrm{eV}$ this dose is minimal, amounting to $\approx 4$ $\mathrm{mC} / \mathrm{cm}^{2}$. Below $E_{p} \approx 80 \mathrm{eV}$ there is a strong increase in dose. Assuming that the hydrogen removal is caused by a direct interaction of the incident electrons with the $\mathrm{Si}-\mathrm{H}$ bond $(\sim 5$ $\mathrm{eV}$ ) via an inelastic scattering process, the efficiency of this process is expected to increase with decreasing electron energy, down to electron energies comparable to typical binding energies. ${ }^{13}$ Just the opposite is seen in Fig. 5. The increase in the dose for $E_{p}<80 \mathrm{eV}$ implies a decrease in efficiency. Therefore, the hydrogen removal cannot be caused by a direct interaction of the incident electrons and the $\mathrm{Si}-\mathrm{H}$ bond. We propose that the hydrogen removal from the silicon surface is caused by secondary electrons emitted from the surface. This is supported by noting that the yield $y$, the number of emitted secondary electrons per incident electron with energy $E_{p}$, is proportional to $E_{p}$, for $E_{p}<100 \mathrm{eV}$ : $y \propto E_{p} .{ }^{14}$ Thus, if secondary electrons are responsible for the hydrogen removal, the dose should approximately increase as $1 / y \propto 1 / E_{p}$ for $E_{p}<100 \mathrm{eV}$. This is what we see in Fig. 5. Additionally, it is noted that secondary electrons have low energies of $\approx 0-10 \mathrm{eV}$ and are therefore expected to have a higher cross section for breaking the $\mathrm{Si}-\mathrm{H}$ bond $(\sim 5 \mathrm{eV})$ than the incident electrons that have higher kinetic energies $E_{p}>20 \mathrm{eV}$. This has important implications for the resolution that can be achieved with hydrogen terminated silicon as $e$-beam resist. The ultimately achievable resolution is estimated, using a simple model to describe this complex process. The resolution will be determined by the surface area from which secondary electrons are emitted. This area is determined by the energy loss per unit of travelled distance of an incident electron $d E / d x$, the stopping power. $d E /$ $d x(E)$ has a maximum of $\approx 10 \mathrm{eV} / \AA$ for electrons of energy $E_{p}=100 \mathrm{eV}^{15}$ For lower energy electrons $d E / d x(E)$ decreases rapidly. Because of this decrease the distance, over which the primary electron has to travel before it has lost its energy, diverges. The electron scattering is approximately isotropic for low energy electrons, as opposed to the high energy electrons that are scattered forward. Therefore it is possible that low energy electrons are scattered below the surface far away from the point of impact of the primary electron. As a consequence, low resolution might be expected. However, the distribution of secondary electrons around the point of impact has to be considered. Since the number of secondary electrons is proportional to the energy loss $d E / d x$, this distribution strongly peaks at the point of impact where $d E / d x$ is maximal. The width of the distribution scales with $E(d E / d x)^{-1}$ is evaluated for $E=E_{p}$, where $E_{p}$ is the energy of the primary electrons; this width is minimal for $E_{p} \approx 100 \mathrm{eV}$ amounting to $2 \mathrm{~nm}$. Higher resolution can only be achieved if the energy of the incident electron is low $\left(E_{p}<10 \mathrm{eV}\right)$ that no secondary electrons are generated, only the primary beam causes the removal of hydrogen.

In the presence of oxygen, exposure of hydrogen terminated $\mathrm{Si}(100)$ to electron-beam radiation results in considerably thicker silicon oxide (see Fig. 4). Figure 6 shows two Auger spectra, one of a locally oxidized area (labelled "exposed"), and the other of an unexposed area (labelled "un-

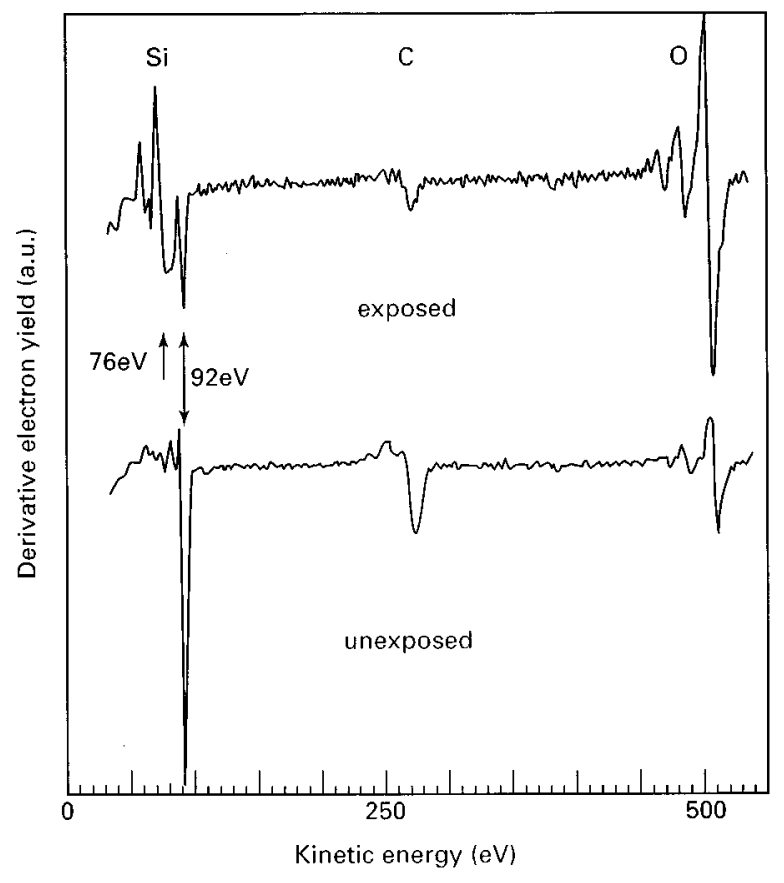

FIG. 6. Auger spectra of an electron-beam exposed area in oxygen environment and an unexposed area on the same substrate $(92 \mathrm{eV}$ is pure silicon peak and $76 \mathrm{eV}$ is chemically shifted silicon oxide peak).

exposed"). The exposed area clearly shows a peak at $92 \mathrm{eV}$, the pure silicon peak from the substrate, and a peak at $76 \mathrm{eV}$, the chemically shifted silicon peak from the oxide layer. On the unexposed area the shifted $\mathrm{SiO}_{2}$ peak is not present. There is a small oxygen peak. This peak presumably comes from the rinse in deionized water after the HF dip. Any fluorine on the surface is then replaced by an $\mathrm{OH}$ group. ${ }^{16}$ From these spectra it can be concluded that $\mathrm{SiO}_{2}$ is formed if a hydrogen terminated Si surface is irradiated with electrons in an oxygen environment.

Figure 7(a) shows the measured oxide thickness as a function of exposure for constant oxygen pressure $\left(10^{-4}\right.$ Torr) and electron energy $(250 \mathrm{eV})$. The rate of oxidation is fast for the first $2 \mathrm{~min}$ (dose of $0.5 \mathrm{C} / \mathrm{cm}^{2}$ ). After longer exposures the oxide thickness saturates at $3 \mathrm{~nm}$. The doses used here are much higher $\left(\sim 1 \mathrm{C} / \mathrm{cm}^{2}\right)$ than the dose required to remove the hydrogen $\left(\sim 0.01 \mathrm{C} / \mathrm{cm}^{2}\right)$. For $1 \mathrm{keV}$ electrons a beam current density of $10 \mathrm{~mA} / \mathrm{cm}^{2}$ (we use a beam current density of $4 \mathrm{~mA} / \mathrm{cm}^{2}$ ) results in a power dissipation of 200 $\mu \mathrm{W}$ in a spot of $50 \mu \mathrm{m}$. This results in a maximum temperature increase $<1 \mathrm{~K}$. It is therefore excluded that heating effects cause the oxidation.

We suggest that the e-beam induced oxidation consist of two steps. The first step is the removal of hydrogen from the surface and the rapid formation of the first silicon oxide monolayer. In the second step the oxidation rate is enhanced by electron exposure. The effect of the electron beam can be twofold: (a) it can enhance the dissociation of adsorbed $\mathrm{O}_{2}$ molecules and (b) it can enhance the diffusion of atomic oxygen into the bulk.

In Fig. 7(b) the dependence of the oxide thickness on the oxygen pressure is shown for $E_{p}=250 \mathrm{eV}$ and an exposure 

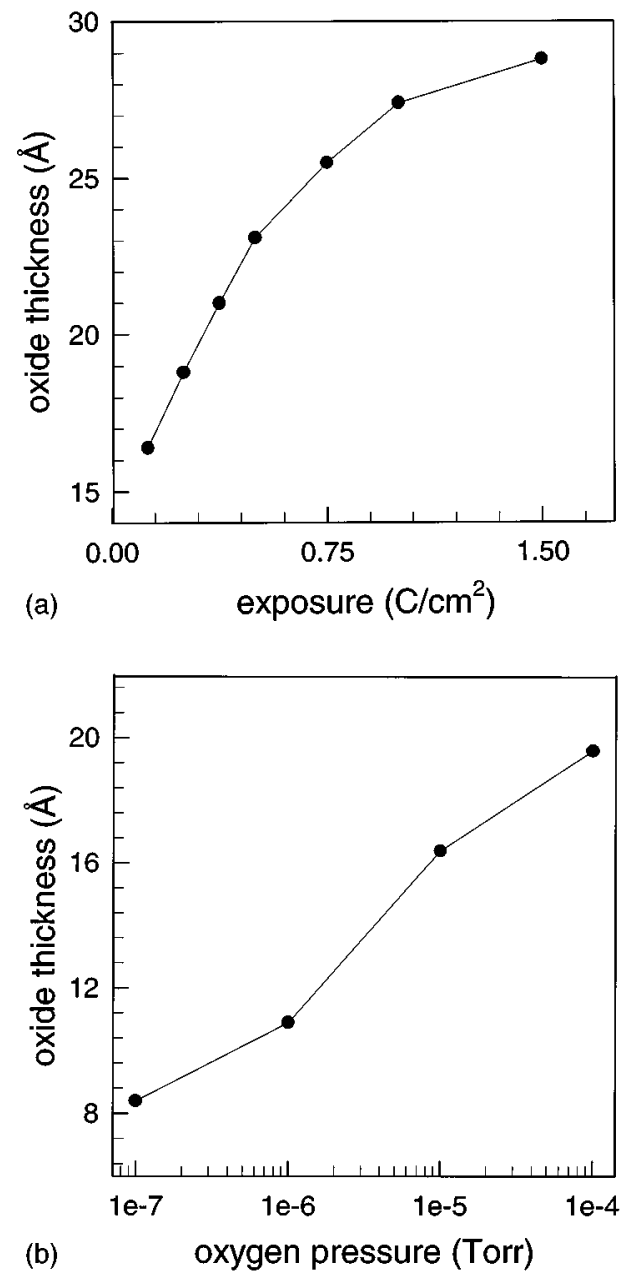

FIG. 7. (a) Oxide thickness as a function of exposure for an electron beam with energy of $250 \mathrm{eV}$ and an oxygen pressure of $10^{-4}$ Torr. (b) Oxide thickness as a function of oxygen pressure for an electron beam with $250 \mathrm{eV}$ and dose of $0.5 \mathrm{C} / \mathrm{cm}^{2}$.

of $0.5 \mathrm{C} / \mathrm{cm}^{2}$. There is an increase in oxide thickness of approximately $2 \AA$ per decade of oxygen pressure. This is not surprising as oxygen is one of the reacting species. A possible mechanism is that oxygen is adsorbed on the surface and then dissociated by impinging electrons. Increasing the oxygen pressure increases the surface coverage; more atomic oxygen can be formed, causing stronger oxidation.

Figure 8 shows the oxide thickness dependence on electron energy for constant dose $\left(0.5 \mathrm{C} / \mathrm{cm}^{2}\right)$ and oxygen pressure $\left(10^{-4}\right.$ Torr). The oxide is thicker for lower energy electrons than for higher energy electrons. This can qualitatively be explained in terms of energy loss of the electrons as they travel within the substrate. The rate of energy loss per unit distance travelled for electrons with $E_{p}>100 \mathrm{eV}$ is approximately inversely proportional to the electron energy, $d E / d x \propto 1 / E .{ }^{14}$ Thus lowering the primary electron beam energy will increase the energy loss near the surface, i.e., increase the oxidation of the surface. Since the higher energy electrons lose most of their energy in the bulk, the oxidation rate on the surface is reduced. This expected dependence is in agreement with the experimental observation in Fig. 8. We believe that the small decrease for $E_{p}<100 \mathrm{eV}$ is due to a

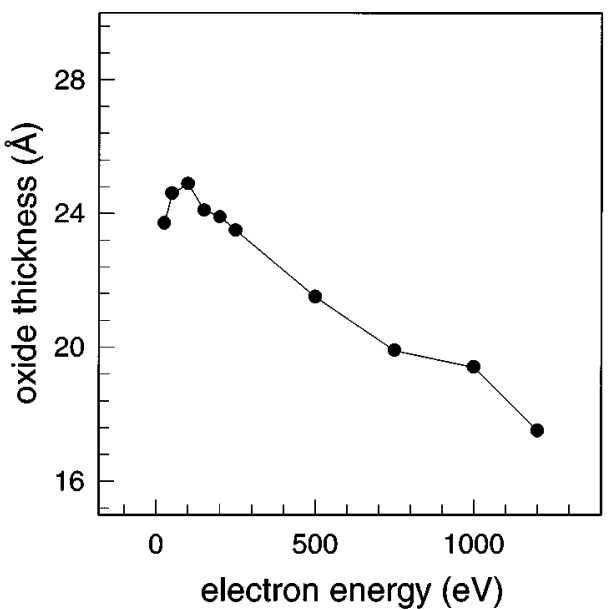

FIG. 8. Oxide thickness as a function of electron energy for an electron dose of $0.5 \mathrm{C} / \mathrm{cm}^{2}$ and oxygen pressure of $10^{-4}$ Torr.

rapidly decreasing $d E / d x$ loss for electrons with kinetic energy $E_{p}<100 \mathrm{eV}$.

\section{CONCLUSIONS AND SUMMARY}

We have studied the oxidation of hydrogen terminated silicon using two techniques: (1) a STM operating in air, and (2) a beam of free electrons in UHV or in a controlled oxygen environment. The mechanism of oxidation with the STM is different than with low energy electrons. Experimental results with the STM are in agreement with an oxidation process that is induced by the electrical field between tip and substrate, rather than by energetic electrons from the tip directly. Experiments with the beam of free electrons shows that in this case the oxidation takes place in two steps. First the hydrogen is removed from the surface, then electron beam enhanced oxidation takes place. The dose for hydrogen removal is minimal for $100 \mathrm{eV}$ electrons, and is $\approx 4 \mathrm{mC} / \mathrm{cm}^{2}$. We have proposed that the removal is caused by secondary electrons emitted from the surface. Based on this, the best resolution that can be obtained with e-beam lithography is estimated to be $\approx 2 \mathrm{~nm}$ for electron energies around $100 \mathrm{eV}$.

From Auger spectra oxide thicknesses between 5 and 30 $\AA$ were measured, depending on electron dose, electron energy, and oxygen pressure. It was argued that lower energy electrons have a higher oxidation rate than higher energy electrons, because the energy loss near the surface is the higher.

We have applied the technique of STM oxidation to pattern $\mathrm{Si}$ and $a$-Si:H using wet etching. Written oxide lines served as an etch mask. With the anisotropic selective etch liquid TMAH, trenches with a width of $35 \mathrm{~nm}$ and a depth of $300 \mathrm{~nm}$ have been made in $\mathrm{Si}(110)$. Oxide lines $(40 \mathrm{~nm})$ were also written on $a-\mathrm{Si}: \mathrm{H}$ and used as an etch mask. $a$-Si:H can be deposited on almost any substrate and is therefore very promising as a high resolution e-beam resist.

\section{ACKNOWLEDGMENTS}

The authors would like to thank J. van Haren, K. Oostveen, and R. in't Ven for STM software and hardware sup- 
port, W. van de Wijgert for the SAM experiments, G. Oversluizen and M. Bouwmans for the $a$-Si:H films, J. Verhoeven for technological assistance, F. Holthuysen for SEM imaging, and J. Jans for the ellipsometry. One author (H.B.) acknowledges financial support from the HCM programme of the EC.

${ }^{1}$ M. A. McCord and R. F. P. Pease, J. Vac. Sci. Technol. B 4, 86 (1986); Y. W. Yau, R. F. W. Pease, A. A. Iranmanesh, and K. J. Polasko, J. Vac. Sci. Technol. 19, 1048 (1981); T. H. P. Chang, D. P. Kern, and L. P. Muray, J. Vac. Sci. Technol. B 10, 2743 (1992); C. R. K. Marrian and E. A. Dobisz, ibid. 10, 2877 (1992); M. A. McCord and T. H. Newman, ibid. 10, 3083 (1992).

${ }^{2}$ J. A. Dagata, J. Schneir, H. H. Haray, C. J. Evans, M. T. Postek, and J. Bennet, Appl. Phys. Lett. 58, 2001 (1990).

${ }^{3}$ J. W. Lyding, T. C. Shen, J. S. Hubacek, J. R. Tucker, and G. C Abeln, Appl. Phys. Lett. 64, 2010 (1994).

${ }^{4}$ E. S. Snow, P. M. Campbell, and B. V. Shanabrook, Appl. Phys. Lett. 63, 3488 (1993).

${ }^{5}$ H. C. Day, and D. R. Allee, Appl. Phys. Lett. 62 (21), 2691 (1993); E. S. Snow and P. M. Campbell, ibid. 64, 1932 (1994).
${ }^{6}$ D. L. Kendall, Appl. Phys. lett. 26, 195 (1975), D. L. Kendall, Annu. Rev. Mater. Sci. 9, 373 (1979).

${ }^{7}$ A. Reisman, M. Berkenblit, S. A Chan, F. B. Kaufman, and D. C. Green, J. Electrochem. Soc. 126, 1406 (1979).

${ }^{8}$ O. Tabata, R. Asahi, H. Funabashi, K. Shimaoka, and S. Sugiyama, Sensors and Actuators A 34, 51 (1992).

${ }^{9}$ N. Hirashita, M. Kinoshita, I. Aikawa, and T. Ajioka, Appl. Phys. Lett. 56, 451 (1990).

${ }^{10}$ C. C. Chang and D. M. Boulin, Surf. Sci. 69, 385 (1977).

${ }^{11}$ M. P. Seah and W. A. Dench, Surf. Interf. Anal. 1, 2 (1979).

${ }^{12}$ T. J. Vink, K. J. B. M. Nieuwesteeg, and G. Oversluizen, J. Appl. Phys. 71, 4399 (1992).

${ }^{13}$ The cross section $\sigma$ for the scattering of fast electrons of kinetic energy $E$ with atoms is approximately proportional to $1 / E$ for $E>\epsilon$, where $\epsilon$ are typical electron energies in the atom. See, for example, Landau and Lifshitz, Course of Theoretical Physics (Pergamon, Great Britain, 1965), vol. 3.

${ }^{14}$ J. L. Goldstein, D. E. Newbury, P. Echlin, D. C. Joy, C. Fiori, and E. Lifsin, Scanning Electron Microscopy and X-ray Microanalysis (Plenum, New York, 1981), pp. 68, 93

${ }^{15}$ C. J. Tung, J. C. Ashley, and R. H. Ritchie, Surf. Sci. 81, 427 (1979).

${ }^{16}$ D. Gräf, M. Grundner, and R. Schulz, J. Vac. Sci. Technol. A 7, 808 (1989). 